\title{
Spitzer Infrared Observations and Independent Validation of the Transiting Super-earth Corot-7 B
}

\section{Citation}

Fressin, Francois, Guillermo Torres, Frederic Pont, Heather A. Knutson, David Charbonneau, Tsevi Mazeh, Suzanne Aigrain, et al. 2011. "SPITZERINFRARED OBSERVATIONS AND INDEPENDENT VALIDATION OF THE TRANSITING SUPER-EARTH CoRoT-7 B." The Astrophysical Journal 745 (1): 81. https://doi.org/10.1088/0004-637x/745/1/81.

\section{Permanent link}

http://nrs.harvard.edu/urn-3:HUL.InstRepos:41397448

\section{Terms of Use}

This article was downloaded from Harvard University's DASH repository, and is made available under the terms and conditions applicable to Other Posted Material, as set forth at http:// nrs.harvard.edu/urn-3:HUL.InstRepos:dash.current.terms-of-use\#LAA

\section{Share Your Story}

The Harvard community has made this article openly available. Please share how this access benefits you. Submit a story.

Accessibility 


\title{
SPITZER INFRARED OBSERVATIONS AND INDEPENDENT VALIDATION OF THE TRANSITING SUPER-EARTH CoROT-7 b
}

\author{
Francois Fressin ${ }^{1}$, Guillermo Torres ${ }^{1}$, Frederic Pont $^{2}$, Heather A. Knutson ${ }^{3}$, David Charbonneau ${ }^{1}$, Tsevi Mazeh $^{4}$, \\ Suzanne Aigrain ${ }^{5}$, Malcolm Fridlund ${ }^{6}$, Christopher E. Henze ${ }^{7}$, Tristan Guillot ${ }^{8}$, and Heike Rauer ${ }^{9}$ \\ ${ }^{1}$ Harvard-Smithsonian Center for Astrophysics, 60 Garden St., Cambridge, MA 02138, USA; ffressin@cfa.harvard.edu \\ ${ }^{2}$ School of Physics, University of Exeter, EX4 4QL Exeter, UK \\ ${ }^{3}$ Astronomy Department, University of California, Berkeley, CA 94720, USA \\ ${ }^{4}$ Department of Astronomy and Astrophysics, Tel Aviv University, 69978 Tel Aviv, Israel \\ ${ }^{5}$ University of Oxford, OX1 3RH Oxford, UK \\ ${ }^{6}$ ESTEC/ESA, P.O. Box 299, 2200 AG Noordwijk, The Netherlands \\ ${ }^{7}$ NASA Ames Research Center, Moffett Field, CA 94035, USA \\ ${ }^{8}$ Observatoire de la Côte d'Azur, BP 4229, 06304 Nice, France \\ ${ }^{9}$ Deutsches Zentrum für Luft und Raumfahrt (DLR), Rutherfordstr. 2, 12489 Berlin, Germany \\ Received 2011 July 18; accepted 2011 October 24; published 2011 December 29
}

\begin{abstract}
The detection and characterization of the first transiting super-Earth, CoRoT-7 b, has required an unprecedented effort in terms of telescope time and analysis. Although the star does display a radial-velocity signal at the period of the planet, this has been difficult to disentangle from the intrinsic stellar variability and pinning down the velocity amplitude has been very challenging. As a result, the precise value of the mass of the planet—and even the extent to which it can be considered to be confirmed — has been debated in the recent literature, with six mass measurements published so far based on the same spectroscopic observations, ranging from about 2 to 8 Earth masses. Here we report on an independent validation of the planet discovery using one of the fundamental properties of a transit signal: its achromaticity. We observed four transits of CoRoT-7 b at $4.5 \mu \mathrm{m}$ and $8.0 \mu \mathrm{m}$ with the Infrared Array Camera (IRAC) on board the Spitzer Space Telescope in order to determine whether the depth of the transit signal in the near-infrared is consistent with that observed in the CoRoT bandpass, as expected for a planet. We detected the transit and found an average depth of $0.426 \pm 0.115 \mathrm{mmag}$ at $4.5 \mu \mathrm{m}$, which is in good agreement with the depth of $0.350 \pm 0.011 \mathrm{mmag}$ (ignoring limb darkening) found by CoRoT. The observations at $8.0 \mu \mathrm{m}$ did not yield a significant detection. The $4.5 \mu \mathrm{m}$ observations place important constraints on the kinds of astrophysical false positives that could mimic the signal. Combining this with additional constraints reported earlier, we performed an exhaustive exploration of possible blend scenarios for CoRoT-7 b using the BLENDER technique. We are able to rule out the vast majority of false positives, and the remaining ones are found to be much less likely than a true transiting planet. We thus validate CoRoT-7 b as a bona fide planet with a very high degree of confidence, independently of any radial-velocity information. Our Spitzer observations have additionally allowed us to significantly improve the ephemeris of the planet, so that future transits should be recoverable well into the next decade. In its warm phase Spitzer is expected to be an essential tool for the validation, along the lines of the present analysis, of transiting planet candidates with shallow signals from CoRoT as well as from the Kepler mission, including potentially rocky planets in the habitable zones of their parent stars.
\end{abstract}

Key words: binaries: eclipsing - planetary systems - stars: individual: CoRoT-7 - stars: statistics - techniques: photometric

Online-only material: color figures

\section{INTRODUCTION}

Among the known exoplanets, a few special cases stand out as the objects that inaugurated the study of the physics of Earth-like exoplanets. CoRoT-7 b (Léger et al. 2009) is the first super-Earth for which the mass and radius have been estimated, and provided the first real constraints on models of the formation, structure, and evolution of small and potentially rocky exoplanets. Kepler- $10 \mathrm{~b}$ has recently been announced as the first rocky planet found by the Kepler mission (Batalha et al. 2011), with a mass of $M_{p}=4.6_{-1.3}^{+1.2} M_{\oplus}$ and a radius of $R_{p}=1.416_{-0.036}^{+0.033} R_{\oplus}$. We now also have examples of interesting planets that are intermediate in mass and radius between the Earth and Neptune, such as GJ $1214 \mathrm{~b}$, with $M_{p}=6.55 \pm 0.98 M_{\oplus}$ (Charbonneau et al. 2009) and $R_{p}=2.64 \pm 0.13 R_{\oplus}$ (Berta et al. 2011).

The first detection of a super-Earth was made possible by the successful CoRoT satellite (Baglin et al. 2002). The discovery was made in the course of observations in the first long run of this mission in the direction of the anti-center of the Galaxy (LRa01), which took place from 2007 October to 2008 March. A small transit-like signal was identified with a depth of $0.35 \mathrm{mmag}$ and a duration of $1.3 \mathrm{hr}$, recurring with a period of 0.8535 days and being consistent with a super-Earth size planet orbiting a bright ( $V=11.7, K=9.8) \mathrm{G} 9$ dwarf star. The discovery triggered a series of follow-up observations to clarify the origin of the shallow transit signal. The detection and follow-up campaign have been fully described by Léger et al. (2009). Bruntt et al. (2010) subsequently performed a detailed spectroscopic analysis of the CoRoT-7 star and determined an improved stellar radius of $R_{\star}=0.82 \pm 0.04 R_{\odot}$. This resulted in a revised planet radius of $R_{p}=1.58 \pm 0.10 R_{\oplus}$.

Queloz et al. (2009) reported on an extensive observational campaign carried out with the HARPS instrument on the ESO $3.6 \mathrm{~m}$ telescope at La Silla, with the goal of detecting the Doppler 
signal of this small object and measuring its mass. CoRoT-7 is an active star, however, with starspot-induced photometric variability at the $\sim 2 \%$ level modulated by the stellar rotation period of about 23 days. The radial velocity of the star is dominated by an irregular signal with an amplitude several times larger than the sought-after signature of the transiting planet. Under these circumstances, measuring the mass accurately is a challenging task that depends strongly on how the activityinduced variability is handled, on assumptions about possible additional non-transiting planets that may be contributing (up to two have been considered), and on the attitude toward systematic errors. Queloz et al. (2009) produced the first mass estimate of $M_{p}=4.8 \pm 0.8 M_{\oplus}$. Subsequent authors have reported different values using the same HARPS observations or subsets thereof. These estimates are not always consistent with each other within their formal errors, and vary considerably in significance level: $6.9 \pm 1.4 M_{\oplus}$ (Hatzes et al. 2010), 2.3 $\pm 1.5 M_{\oplus}$ (Pont et al. 2011), $8.0 \pm 1.2 M_{\oplus}$ (Ferraz-Mello et al. 2011), $5.7 \pm 2.5 M_{\oplus}$ (Boisse et al. 2011), and $7.4 \pm 1.2 M_{\oplus}$ (Hatzes et al. 2011). The impact of the discrepancies is not insignificant, as these estimates lead to rather different mean densities and therefore different internal structures for the planet as inferred from current theory. While some of the more recent determinations appear to favor a higher mass for the planet, at the $2 \sigma$ level the estimates range all the way from 0 to $10 M_{\oplus}$, and for some of the lower values (Pont et al. 2011; Boisse et al. 2011) the statistical significance of the Doppler detection of CoRoT-7 b is considerably less compelling.

Similar difficulties are expected to occur for other candidates with shallow transits, in which "confirmation" in the usual sense of the word by the detection of a radial-velocity signature that is at the limit of detectability with current instrumentation will be very challenging. With the recent announcement of a large number of shallow transit candidates discovered by the Kepler satellite (Borucki et al. 2011), obtaining assurance that these signals correspond to bona fide super-Earth size planets, as opposed to a false positive, is among the most urgent tasks that lie ahead, and is of primary importance for the statistical interpretation of the results.

Motivated by the lingering problems with the CoRoT-7 b mass determination described above, and the implications for the robustness of the detection, we use this case here to illustrate the application of a powerful technique to help "validate"10 shallow transit candidates independently of any radial-velocity information. It makes use of near-infrared observations with the Spitzer Space Telescope, and is based on the premise that true transits are achromatic signals, to first order, so that the transit depth as observed in the near-infrared should be the same as in the CoRoT passband (ignoring the effects of limb darkening). If the candidate is the result of a blend, however, the depth can be significantly different. As an example, if the observed transit were due to an object eclipsing a background star of $0.5 M_{\odot}$ and sun-like age and metallicity, then its transit in the Spitzer $4.5 \mu \mathrm{m}$ bandpass would be 3.2 times deeper than in the CoRoT bandpass. With its infrared passband, Spitzer affords the maximal wavelength separation from the CoRoT photometry (passband around $650 \mathrm{~nm}$ ), and places very strong constraints on possible false positive scenarios, as we describe below.

\footnotetext{
10 In the context of this paper, "confirmation" as used above refers to the unambiguous detection of the gravitational influence of the planet on its host star (e.g., the Doppler signal) to establish the planetary nature of the candidate; when this is not possible, we speak of "validation," which involves an estimate of the false alarm probability.
}

Even with these constraints, and others available from followup observations carried out and reported by the CoRoT team, it is not possible to rule out all possible blend configurations for CoRoT-7 b, as also recognized by Léger et al. (2009). Thus, the main goal of this work is to more exhaustively explore the wide variety of false positive scenarios that can mimic the light curve to obtain a realistic estimate of the blend frequency (BF) that may be expected. We aim to provide an independent assessment of the confidence level that the signal is of planetary nature. Rather than focusing solely on the likelihood of a blend (frequentist approach), as in previous studies, we adopt a Bayesian approach in which we compare the BF with a prior for the likelihood of a planet (odds ratio). To evaluate the BF we make use of the BLENDER technique introduced by Torres et al. (2004, 2011), with further developments as described by Fressin et al. (2011). This methodology has been applied successfully to validate a number of shallow transit signals from the Kepler mission including Kepler-9 d, Kepler-10 c, Kepler-11 g, and Kepler-19 b (Torres et al. 2011; Fressin et al. 2011; Lissauer et al. 2011; Ballard et al. 2011).

We begin by describing our Spitzer observations (Section 2), and then briefly summarizing the data used here along with other follow-up observations relevant to this investigation (Section 3). This is followed by the BLENDER analysis that examines the vast space of parameters for false positives by synthesizing realistic blend light curves and comparing them with the CoRoT photometry (Section 4). We next estimate the expected frequency of blends and compare it with the expected frequency of planets (Section 5). As shown below, this analysis is able to validate CoRoT-7 b as a planet without relying on any radialvelocity information.

\section{SPITZER OBSERVATIONS OF CoRoT-7 b}

The Infrared Array Camera (IRAC; Fazio et al. 2004) of the Spitzer Space Telescope (Werner et al. 2004) obtains simultaneous images in four bandpasses. A $5.2 \times 5.2$ field of view (FOV) is imaged in one pair of bandpasses $(3.6$ and $5.8 \mu \mathrm{m})$ and a nearly adjacent FOV imaged in the second pair ( 4.5 and $8.0 \mu \mathrm{m})$. The two blue channels employ InSb detectors, whereas the red channels use Si:As IBC detectors. All four arrays are $256 \times 256$ pixels. While the present Warm Spitzer mission is restricted to the two shorter wavelengths, the data discussed in this section were obtained just prior to the spacecraft entering that phase in May of 2009. We elected to monitor CoRoT-7 in only one channel pair $(4.5$ and $8.0 \mu \mathrm{m})$, as even if the stellar flux in these bandpasses is slightly smaller, we have obtained more precise observations in the past using this pair (Knutson et al. 2008; Fressin et al. 2009). It is also farther in wavelength from the CoRoT bandpass, and the expected depth difference if the signal comes from a blend scales with the wavelength difference, as we describe later.

We used IRAC to observe the primary eclipse of CoRoT-7 b on UT 2009 April 22, 23, 24, and 25, obtaining data at 4.5 and $8.0 \mu \mathrm{m}$. We were able to observe it in full array mode in both channels for a total duration of $17.0 \mathrm{hr}$, including four transits lasting 75 minutes each. We observed the target in the IRAC stellar mode, in which the camera gathers simultaneously two $10.4 \mathrm{~s}$ integrations at 4.5 and $8.0 \mu \mathrm{m}$. Therefore, we gathered, respectively, $1075,1212,1212$, and 1212 images both at 4.5 and $8.0 \mu \mathrm{m}$ during the four transits we observed. Our goal was to detect the transit signal at $4.5 \mu \mathrm{m}$, but as the $8.0 \mu \mathrm{m}$ observations are simultaneous and automatic, we present them hereafter for completeness. We describe below our observations 
in two sections, as the InSb detectors used for IRAC channels at $4.5 \mu \mathrm{m}$ require a different treatment than the $\mathrm{Si}$ :As detectors of the IRAC $8.0 \mu \mathrm{m}$ channel.

We were mindful that the signal we were looking for was at the limit of what was possible to obtain with a few transits from Spitzer, as it has mainly been used to look at brighter stars and deeper eclipses. Our expectation in terms of statistical significance for the detection of a single transit was $2.1 \sigma$ at $4.5 \mu \mathrm{m}$ ( $4.2 \sigma$ when combining the four transits), scaled on the detection level we achieved in our previous studies of TrES-1, TrES-3, and TrES-4 (Charbonneau et al. 2008; Knutson et al. 2008; Fressin et al. 2009).

\section{1. $4.5 \mu \mathrm{m}$ Observations}

The contribution of the background to the total flux from CoRoT-7 is low in the $4.5 \mu \mathrm{m}$ IRAC bandpass, contributing only $0.35 \%$ to the total flux in an aperture with a 5 pixel diameter centered on the position of the star. We obtained the lowest rms time series using an aperture with a radius of 5.0 pixels. We allowed the position of our aperture to shift with the position of the star in each image. We estimated the background in each image from an annulus with an inner radius of 12 pixels and an outer radius of 20 pixels centered on the position of the star.

We determined the position of the star in each image by fitting a two-dimensional Gaussian to the position of the star. Agol et al. (2010) have recently completed a comparative analysis of different methods to estimate the stellar centroid in Spitzer images. Their best results are obtained by fitting a twodimensional Gaussian to the star's point-spread function (PSF), compared to a flux-weighted centroiding, and parabolic fitting. This Gaussian algorithm uses the $7 \times 7$ pixel sub-array from the image centered on the brightest pixel. It fits a two-dimensional Gaussian to this array, allowing its center, amplitude, and width to vary. It then uses a nonlinear Levenberg-Marquardt algorithm to optimize these parameters (Markwardt 2009). We compared this technique with the position-weighted sum of the flux in a 4 pixel radius disk centered on the approximate position of the star, which we previously used on similar magnitude targets (Charbonneau et al. 2008; Knutson et al. 2008; Fressin et al. 2009). The Gaussian fit proved to be better regarding two criteria. First, the scatter between the position estimates was 1.3 times smaller. Second, the scatter of the differential position between our target star and a nearby reference star ( 2 mag fainter in channel 2 and at $28^{\prime \prime}$ ) was smaller and did not show any correlation with the intra-pixel position.

The dominant instrumental systematic effect in the first two IRAC bandpasses is due to a well-known intra-pixel sensitivity (Reach et al. 2005; Charbonneau et al. 2005, 2008; MoralesCalderon et al. 2006; Knutson et al. 2008). Fluxes at these two wavelengths show a strong correlation with the intra-pixel position of the star on the detector, at a level comparable to the expected depth of the eclipse. We used the following parameters to fit the observed flux as a linear function of the subpixel position:

$$
f=f_{0} *\left[c_{1}+c_{2}\left(x-x_{0}\right)+c_{3}\left(y-y_{0}\right)\right],
$$

where $f_{0}$ is the original flux from the star, $f$ is the measured flux, $x$ and $y$ denote the location of the Gaussian-fit centroid of the star on the array, $x_{0}$ and $y_{0}$ are the coordinates of the center of the pixel containing the peak of the star's PSF, and $c_{1}-c_{3}$ are free parameters in the fit. We excluded the in-transit measurements, based on the known ephemeris, in order to avoid suppressing the transit depth. For the $x$ and $y$ positions above we calculated the centroid of the target in each image and found that the pointing jitter was around 0.12 pixels $(0.14)$ over the course of a visit in both $x$ and $y$. The pointing drift of the telescope appears to occur on a longer timescale than the exposures. Therefore, instead of using the actual position estimate at individual exposure times, we smoothed the $x$ and $y$ curves as a function of time and used the smoothed position instead.

In contrast to previous observations of HD 189733 and HD 209458 (Knutson et al. 2008; Charbonneau et al. 2008), we found that adding quadratic or higher-order terms to this equation, or even cross-terms, did not improve the fit significantly, likely due to the lower signal-to-noise ratio of the present observations.

After correcting for the intra-pixel sensitivity, a decreasing trend was still visible that is likely to be an instrumental effect related to the detector or telescope, and has also been seen in observations of TrES-3 and TrES-4 (Knutson et al. 2008; Fressin et al. 2009), two stars with similar brightness. We corrected for this effect by fitting the data in both channels with a linear function of time. This term was fitted simultaneously with the correction for the intra-pixel sensitivity, so that we can accurately characterize the additional uncertainty in the depth and timing of the eclipse introduced by these corrections. That is to say, we solved for four parameters including a constant term, a linear function of $x$ position, a linear function of $y$ position, and a linear function of time. We also trimmed the first 30 minutes of data that show a larger scatter, as we have done in previous cases. The fit was performed with a Markov chain Monte Carlo (MCMC) method (Ford 2005; Winn et al. 2007) with $5 \times 10^{5}$ steps, where we set the uncertainty of the individual measurements equal to the standard deviation of the out-oftransit data after correction for the various detector effects.

Prior to the fit we carried out an initial trimming within our aperture, discarding outliers farther than $3.5 \sigma$ from the local median flux (defined as the median of a 15 minute window centered on the data point). We also removed measurements for which the identified position of the photocenter $x$ or $y$ deviated by more than $3.5 \sigma$ from the same 15 minute median position. This global trimming excludes $6.6 \%$ of the data points in the four visits in the $4.5 \mu \mathrm{m}$ bandpass. We excluded outliers greater than $3.5 \sigma$ during each step of the chain, as determined using the residuals from the model light curve, from our evaluation of the $\chi^{2}$ function. We rescaled the value of the $\chi^{2}$ function to account for the fact that we are varying the number of pixels included in the fit.

After producing the chain, we searched for the point in the chain where the $\chi^{2}$ value first falls below the median of all the $\chi^{2}$ values (i.e., where the code had first found the best-fit solution) and discarded all the steps up to that point. Figure 1 shows the four individual light curves and the respective fits for the instrumental effects that we removed from these curves before looking for the transit signal itself.

Next we carried out a second Markov chain fit of the transit signal itself on the trimmed data. We initially allowed the individual depths and times of the four transits to vary independently, along with the normalized semimajor axis $a / R_{\star}$ and the inclination angle of the orbit. We calculated our transit curves using the formulation by Mandel \& Agol (2002). Although small at $4.5 \mu \mathrm{m}$, limb darkening was taken into account in our modeling according to the four-parameter law by Claret (2000), with coefficients taken from the work of Sing (2010) and stellar parameters $T_{\text {eff }}=5250 \pm 60 \mathrm{~K}$, 


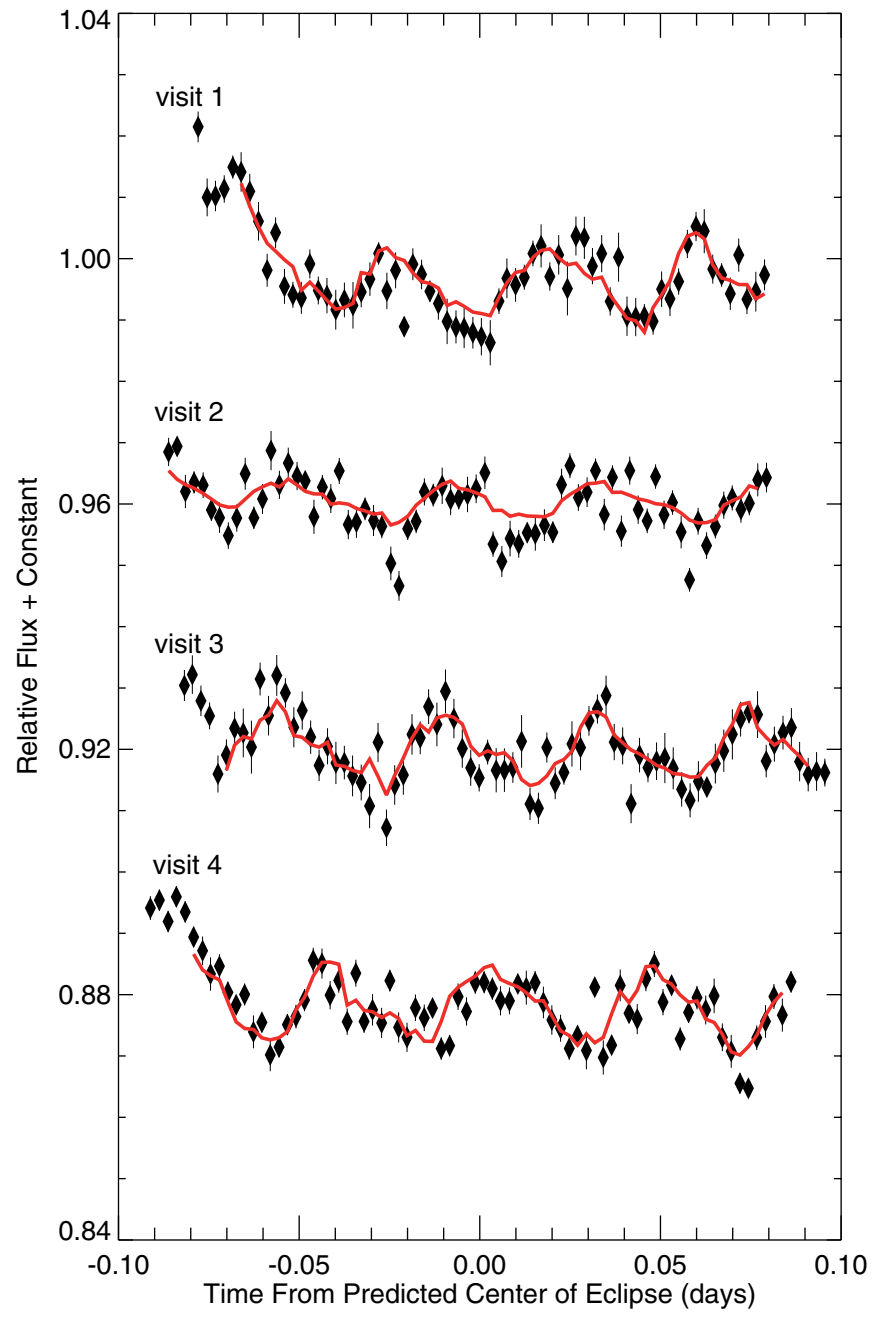

Figure 1. Transit light curves of CoRoT-7 b in channel $2(4.5 \mu \mathrm{m})$ observed on UT 2009 April 22, 23, 24, and 25, with best-fit curves representing instrumental effects overplotted in red. Data have been binned in 7.3 minute intervals, then offset by a constant for the purposes of this plot.

(A color version of this figure is available in the online journal.)

Table 1

Best-fit Transit Parameters

\begin{tabular}{lcc}
\hline \hline Parameter & CoRoT $^{\mathrm{a}}$ & Spitzer $4.5 \mu \mathrm{m}$ \\
\hline$P$ (days) & $0.853585 \pm 0.000024$ & $0.853590 \pm 0.000006$ \\
$a / R_{\star}$ & $4.27 \pm 0.20$ & $4.1_{-1.6}^{+2.4}$ \\
Inclination (deg) & $80.1 \pm 0.3$ & $83.6_{-8.3}^{+6.4}$ \\
Depth (mmag) & $0.350 \pm 0.011$ & $0.426 \pm 0.115$ \\
\hline
\end{tabular}

Note. ${ }^{\text {a }}$ Léger et al. (2009).

$\log g=4.47 \pm 0.05$, and $[\mathrm{Fe} / \mathrm{H}]=+0.12 \pm 0.06$ from Bruntt et al. (2010). While the individual depths and transit time parameters were consistent with transits occurring at the expected period from the CoRoT light curve, the signal-to-noise ratios were too poor to provide a meaningful constraint on the geometric parameters. We therefore chose to restrict the space of free parameters for the Markov chain analysis to the transit depth, $a / R_{\star}$, inclination angle, and period, and we held the transit epoch fixed at the value reported by Léger et al. (2009).

Non-random sources of noise in transit and eclipse photometry—such as instrumental systematics and stellar variability - could dominate the error budget in the derived

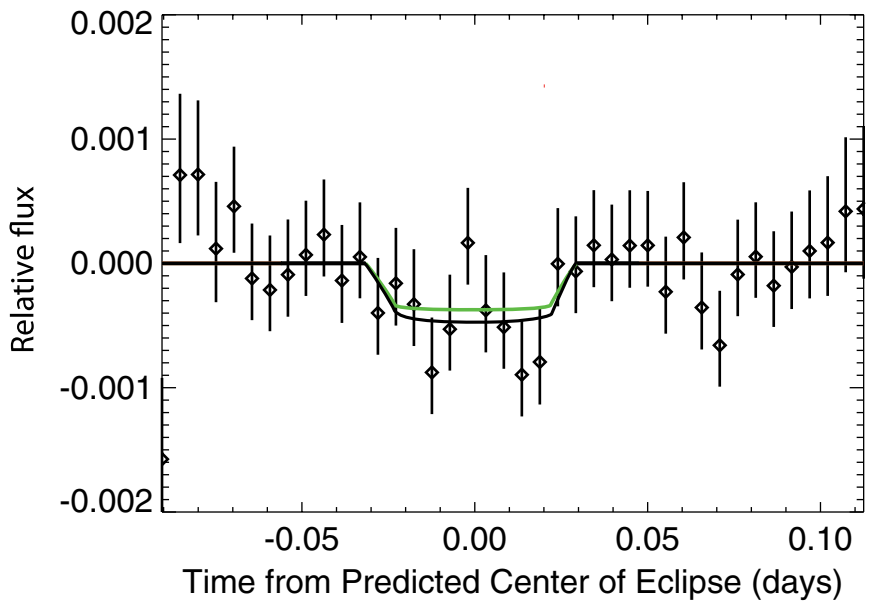

Figure 2. Transit light curve of CoRoT-7 b in the IRAC $4.5 \mu \mathrm{m}$ bandpass after removal of instrumental effects, folded on the expected period. Data have been normalized to remove detector effects (see discussion in Section 2.1), and binned in 9.2 minute intervals. The black curve is the best transit model fit to the Spitzer light curve, imposing the CoRoT ephemeris. The transit depth is $0.426 \pm 0.115 \mathrm{mmag}$. The green curve shows the model expected for the super-Earth transit scenario, adopting the CoRoT parameters from Léger et al. (2009), which lead to a depth of $0.350 \pm 0.011 \mathrm{mmag}$. The good agreement in the depth indicates that the transit is achromatic, as expected for a planet.

(A color version of this figure is available in the online journal.)

system parameters. This is true for ground-based data and also turns out to be true for space-based data. The higher stability of space measurements is offset by the fact that smaller effects are being measured and correspondingly smaller levels of random error are achieved by collecting more signal. Pont et al. (2006) showed how neglecting this type of noise could lead to an underestimate of the actual uncertainties by a large factor. In the present study, a realistic error estimate is important and we therefore attempted to assess the possible impact of non-random noise. The presence of correlated noise is obvious in our raw photometric sequence. We corrected for it to first order, but obviously the correction cannot be perfect. We used the singleparameter description of the correlated component of the noise proposed by Pont et al. (2006), with the further simplification of Winn et al. (2009) adapted to regularly sampled data, to estimate the impact of the residual systematics after decorrelation. We repeated the MCMC analysis by using modified uncertainties $\sigma_{\text {tot }}^{2}=\sigma_{\mathrm{w}}^{2}+n \sigma_{\mathrm{r}}^{2}$, where $\sigma_{\mathrm{w}}^{2}$ and $\sigma_{\mathrm{r}}^{2}$ are the "white" and "red" components of the noise (i.e., random and correlated, respectively), and $n$ is the number of data points during the eclipse. We estimated $\sigma_{\mathrm{r}}$ from the dispersion of the flux after decorrelation obtained with slightly different, reasonable decorrelation procedures. We used $\sigma_{r}=0.000108 \mathrm{mag}$ as the dispersion between three different decorrelation techniques (i.e., fitting the intrapixel answer and the transit simultaneously, adding quadratic terms to the intra-pixel answer, and the decorrelation technique previously described).

Table 1 collects our results, and the Spitzer time series is shown in Figure 2 along with the fitted model. The parameters we derive for the planet are in good agreement with those based on the light curve as observed by the CoRoT satellite. In particular, the near-infrared and optical depths are consistent within the errors. Also shown in Figure 2 is a model based on the optical light curve parameters for comparison with the Spitzer curve. Although we do not reach the precision Léger et al. (2009) obtained for the geometric parameters based on their detection of 153 individual transits, we were able to improve the precision 
in the period determination of the planet significantly, as our Spitzer observations were gathered some 640 planetary orbits after the original CoRoT data.

To put our results on a more quantitative basis, we computed the reduced $\chi^{2}$ values corresponding to the case where no transit at all is shown in the data, and the case of a transit with a depth corresponding to a planet such as CoRoT-7 b. We find that the super-Earth scenario gives a reduced $\chi^{2}(1.0039)$ very close to the best fit to the data (1.0033), whereas the no-transit scenario provides a poor fit (1.0276).

Since the transit depth we measure in the near-infrared is about the same as in the optical, this argues against blends composed of stars of much later spectral type. Importantly, we can use the error bar on the measured depth to rule out blends involving stars of a different temperature that would have produced a significantly different depth in infrared. Indeed, the depth of a blend in the Spitzer bandpass compared to the optical depends solely on the infrared contribution of the blending star to the total flux relative to its contribution in the CoRoT bandpass. For example, if the relative contribution in the infrared of a background eclipsing binary is three times larger in infrared than in the visible, then the observed transit in the infrared would be three times deeper. The relative infrared-to-visible contribution of a background star is also independent of its distance to the target to first order (i.e., ignoring the effects of interstellar dust). As a consequence, for a given target star, the observed depth increase or decrease of a blend when observed in the Spitzer bandpass is only a function of the spectral type of the contaminating star. Using an isochrone of solar age and metallicity representative of the background stars in the Galactic plane, it is straightforward to compute the change in the relative infrared-to-visible flux contribution of such a star as a function of its mass, and hence the depth increase or decrease in the 4.5 and $8 \mu \mathrm{m}$ bandpasses that a blend would display. With the target properties (mass $M=0.91 M_{\odot}$, age $\approx 2$ Gyr) from Bruntt et al. (2010), we find for CoRoT-7 that all blended stars below $0.69 M_{\odot}$ would show a transit deeper than the $3 \sigma$ upper limit observed with Spitzer at $4.5 \mu \mathrm{m}(0.77 \mathrm{mmag})$. We make use of this important constraint later in Section 5 to eliminate a large fraction of potential false positives for CoRoT-7 b.

\section{2. $8.0 \mu \mathrm{m}$ Observations}

For the observations at $8.0 \mu \mathrm{m}$ we used the "preflash" technique (Knutson et al. 2009), in which we pointed the telescope for 30 minutes toward a bright $\mathrm{H}$ II region before observing CoRoT-7. This was completed in order to reduce the amplitude of the detector "ramp" at $8.0 \mu \mathrm{m}$, effectively preloading the pixels on which the target star would be pointed.

Previous secondary eclipse studies (e.g., Knutson et al. 2008) have shown that PSF fitting can provide a better signal-to-noise ratio at longer wavelengths. At longer wavelengths the flux from the star is smaller and the zodiacal background is larger; we find that the background contributes $20 \%$ of the total flux in a 3 pixel aperture at $8.0 \mu \mathrm{m}$.

At $8.0 \mu \mathrm{m}$, we found that the relative scatter in the time series after model fitting from the PSF fits was $15 \%$ higher than in the time series from aperture photometry with a 3.0 pixel radius. As a result of this increased scatter, which is likely produced by discrepancies between the model PSF and the observed PSF, we concluded that aperture photometry is also preferable. We compared the time series using apertures ranging from 3.0 to 4.5 pixels and found consistent results in all cases, but with a scatter that increases with the radius of the photometric aperture.

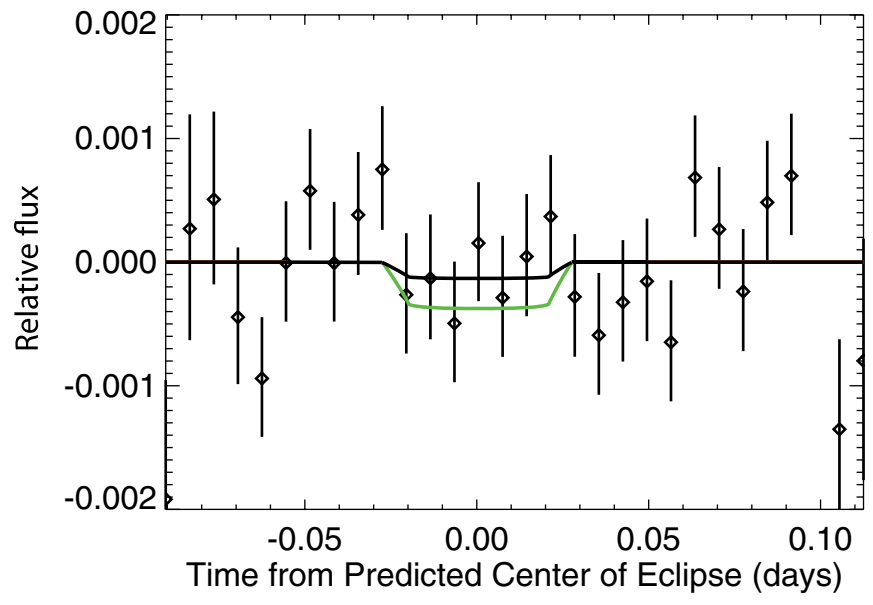

Figure 3. Transit light curve of CoRoT-7 b in the IRAC $8.0 \mu \mathrm{m}$ bandpass after removal of instrumental effects, phased with the expected period and with the best-fit transit curve overplotted (see the text). Data have been normalized to remove detector effects (see discussion in Sections 2.1 and 2.2), and binned in 9.2 minute intervals. The black curve represents the best transit model fit adopting the CoRoT ephemeris, which gives a statistically insignificant depth of $0.11 \pm 0.30 \mathrm{mmag}$. The green curve shows the fit expected for the super-Earth transit scenario, using the parameters from Léger et al. (2009).

(A color version of this figure is available in the online journal.)

Previous observations (e.g., Knutson et al. 2009) have shown that the ramp is well described as following an asymptotic shape, with a steeper rise in the first 30 minutes of observations. We corrected for this effect by fitting our time series with the following function:

$$
f=f_{0} *\left[c_{1}+c_{2} \ln (d t)\right],
$$

where $f_{0}$ is the original flux from the star, $f$ is the measured flux, and $d t$ is the elapsed time in days since the start of the observations.

As the expected statistical significance of the signal at $8.0 \mu \mathrm{m}$ was very small (i.e., $1.1 \sigma$ ), we chose to fix the transit time and period of the transit to values found in our $4.5 \mu \mathrm{m}$ study and verified that the residual signal in the four visits at $8.0 \mu \mathrm{m}$ was compatible with the transiting planet scenario. We carried out an MCMC fit to the data as described in Section 2.1, simultaneously fitting Equation (2) and a transit model, with the depth as the only free parameter in the latter. No significant correlations were found between the variables. As a further check we repeated these fits adding a quadratic term of the form $\ln (d t)$ in Equation (2) and found that the value of $\chi^{2}$ for our best-fit solution was similar to the previous value, so we chose not to include this additional term.

Figure 3 shows the detrended (from the ramp), trimmed, binned, and folded channel 4 light curve. We estimated the best-fit transit depth using an MCMC fit in the same way as we did for the $4.5 \mu \mathrm{m}$ data and found an eclipse depth of $0.11 \pm 0.30 \mathrm{mmag}$. The noise level is too large to say anything about a dip corresponding to the planet eclipse, but it disallows a blend scenario involving a significantly redder star. Specifically, proceeding in the same way as described at the end of Section 2.1, a star of $0.5 M_{\odot}$ would be excluded at the $3 \sigma$ level.

\section{CORoT PHOTOMETRY AND FOLLOW-UP OBSERVATIONS}

The photometric observations used in the next section to investigate blends are essentially the same as used by Léger 
et al. (2009), with a somewhat different detrending of the data. We started with the N2 (science grade) light curve, discarding the initial few days, which had a time sampling of $512 \mathrm{~s}$, and worked only with the remainder, where the time sampling was 32 s (see Léger et al. 2009 for a detailed description of the N2 light curve). We identified and clipped outliers using a running five-point median filter and then modeled the intrinsic stellar variability using an iterative nonlinear filter (Aigrain \& Irwin 2004). This consists in applying a five-point boxcar filter followed by a median filter with a width of 1 day, $3 \sigma$ clipping the residuals, and iterating until no more points are clipped. The resulting slowly varying component was then subtracted from the original light curve to give the time series used in this work. We made use of the white-light data only. Of the extensive follow-up observations carried out by the CoRoT team as described by Léger et al. (2009), the most relevant for our study are the high-resolution imaging observations obtained with the NACO instrument on the Very Large Telescope (VLT). These data exclude any blends capable of mimicking the signal outside of 0.'4 from the target down to 6.5 mag fainter than the target. Additionally, the spectroscopic observations reported by these authors allowed them to rule out companions that would be bright enough to be visible in the spectrum (as a second set of lines). In particular, their near-infrared ( $K$-band) spectroscopy with CRIRES on the VLT rules out most companions brighter than about $7 \%$ of the flux of the target in that band $(\Delta m \approx 2.9)$. For the analysis below we adopt a more conservative limit of $\Delta m=1.0$.

\section{BLENDER ANALYSIS}

Blend scenarios satisfying the CoRoT observations were explored with the BLENDER technique (Torres et al. 2004, 2011; Fressin et al. 2011) by examining the quality of the fit to the CoRoT-7 photometry of a large array of synthetic model light curves. These synthetic light curves result from the combined light of three objects composing the blend: the main target, and an unresolved eclipsing pair along the line of sight with the same period as detected in CoRoT-7, whose eclipses are attenuated by the light from the target. The eclipsing system may be physically associated (hierarchical triple), or may be in the background or foreground, and the eclipsing pair may consist of two stars, or a star transited by a larger planet. BLENDER uses the detailed shape of the transit light curve to weed out scenarios that lead to the wrong shape for a transit. The properties of the three objects were taken from model isochrones by Girardi et al. (2000) in order to synthesize light curves, and these artificial light curves were compared with the observations in a $\chi^{2}$ sense (i.e., by computing the sum of the squared residuals normalized by the photometric uncertainties). For practical reasons, blend light curves were calculated here in the Kepler passband, which is sufficiently similar to the passband of the CoRoT satellite for our purposes, in both the central wavelength and width. ${ }^{11}$ The properties of the main star (essentially its intrinsic brightness, which affects the dilution of the eclipses) were constrained by the spectroscopic observations of CoRoT-7 $\left(T_{\text {eff }}=5250 \pm\right.$ $60 \mathrm{~K}, \log g=4.47 \pm 0.05,[\mathrm{Fe} / \mathrm{H}]=+0.12 \pm 0.06$; Queloz et al. 2009; Bruntt et al. 2010) and held fixed. Those of the other two objects (referred to here as the "secondary" for the eclipsed star and "tertiary" for the eclipsing object) were varied over a wide range and parameterized by mass. For hierarchical triples the stellar components were constrained to lie on the same

\footnotetext{
11 See http://keplergo.arc.nasa.gov/CalibrationResponse.shtml.
}

isochrone, while for background/foreground blends the properties of the intruding star were taken from a representative solar-metallicity, 3 Gyr isochrone.

The distance between the binary $\left(d_{\mathrm{EB}}\right)$ and the target $\left(d_{\text {CoRoT-7 }}\right)$ was expressed for convenience in terms of the distance modulus difference $\left(\Delta \delta=5 \log \left[d_{\mathrm{EB}} / d_{\text {CoRoT-7 }}\right]\right)$, which is independent of interstellar extinction, and varied between -5 and +10 . The impact parameter was allowed to vary between 0 and unity, and the mass of the secondary stars ranged from $0.1 M_{\odot}$ to $1.4 M_{\odot}$. For stellar tertiaries we explored masses from $0.1 M_{\odot}$ up to the mass of the secondary; for tertiaries that are planets (assumed to be dark) we allowed their sizes to be up to $2.0 R_{\text {Jup }}$. We restricted our blend simulations to circular orbits for all eclipsing pairs, as no stellar or planetary systems with periods as short as that of CoRoT-7 $b$ are known to have eccentric orbits, nor are expected to be from theoretical arguments (see, e.g., Mazeh 2008). Synthetic light curves were generated with a detailed eclipsing binary code, including proximity effects (tidal and rotational distortions), limb darkening, gravity brightening, and contamination from third light. Differential extinction was included for chance alignments.

Blends providing poor fits compared to the CoRoT-7 photometry in a $\chi^{2}$ sense were considered to be ruled out. This enables us to place constraints on the kinds of objects composing the eclipsing pair that yield viable blends (i.e., acceptable fits), including their size or mass, as well as other properties of the blend such as the overall brightness and color. For further details and applications of BLENDER to other transiting planet candidates, we refer the reader to the work of Torres et al. (2011), Lissauer et al. (2011), and Fressin et al. (2011).

\subsection{Background or Foreground Stars Transited by a Planet}

We consider first the case of blends involving a background or foreground star falling within the CoRoT aperture and transited by a larger planet. Because of the very short 0.8535 day orbital period of CoRoT-7, evolved stars (giants or subgiants) are ruled out, so we focus in the following on main-sequence stars. We find that there is a very large range of spectral types (masses) permitted for the secondary star, shown in the top panel of Figure 4, as well as a wide range of relative distances between the eclipsing pair and the target. This is indicated by the darker areas in the figure, delimited by the white contour representing light curve fits that differ from the best transiting planet model by a $\chi^{2}$ difference corresponding to a $3 \sigma$ confidence level. However, other constraints available for CoRoT-7 strongly restrict the number of these false positives. This is illustrated in the bottom panel of the figure, in which these additional constraints are superposed on the same blend landscape displayed in the top panel. In particular, by comparing the predicted $r-K_{s}$ color of each blend against the measured color of the star from Exodat $\left(r-K_{s}=1.723 \pm 0.025\right.$; Léger et al. 2009), we find that a large fraction of the secondaries with significantly different masses than the primary are ruled out because the blends would be too red or too blue compared to the known color index of CoRoT-7 (by more than $3 \sigma$ ). These excluded regions are indicated by the blue hatched areas in the bottom panel of Figure 4. Other blends are excluded because the secondary star would be very bright (within 1 mag of the target) and would have been noticed spectroscopically, if unresolved in the high-resolution imaging described earlier. The section of parameter space excluded by this brightness criterion is indicated by the green hatched area, limited from above by the green diagonal line corresponding to a magnitude difference of $1.0 \mathrm{mag}$. Note that, as mentioned in 

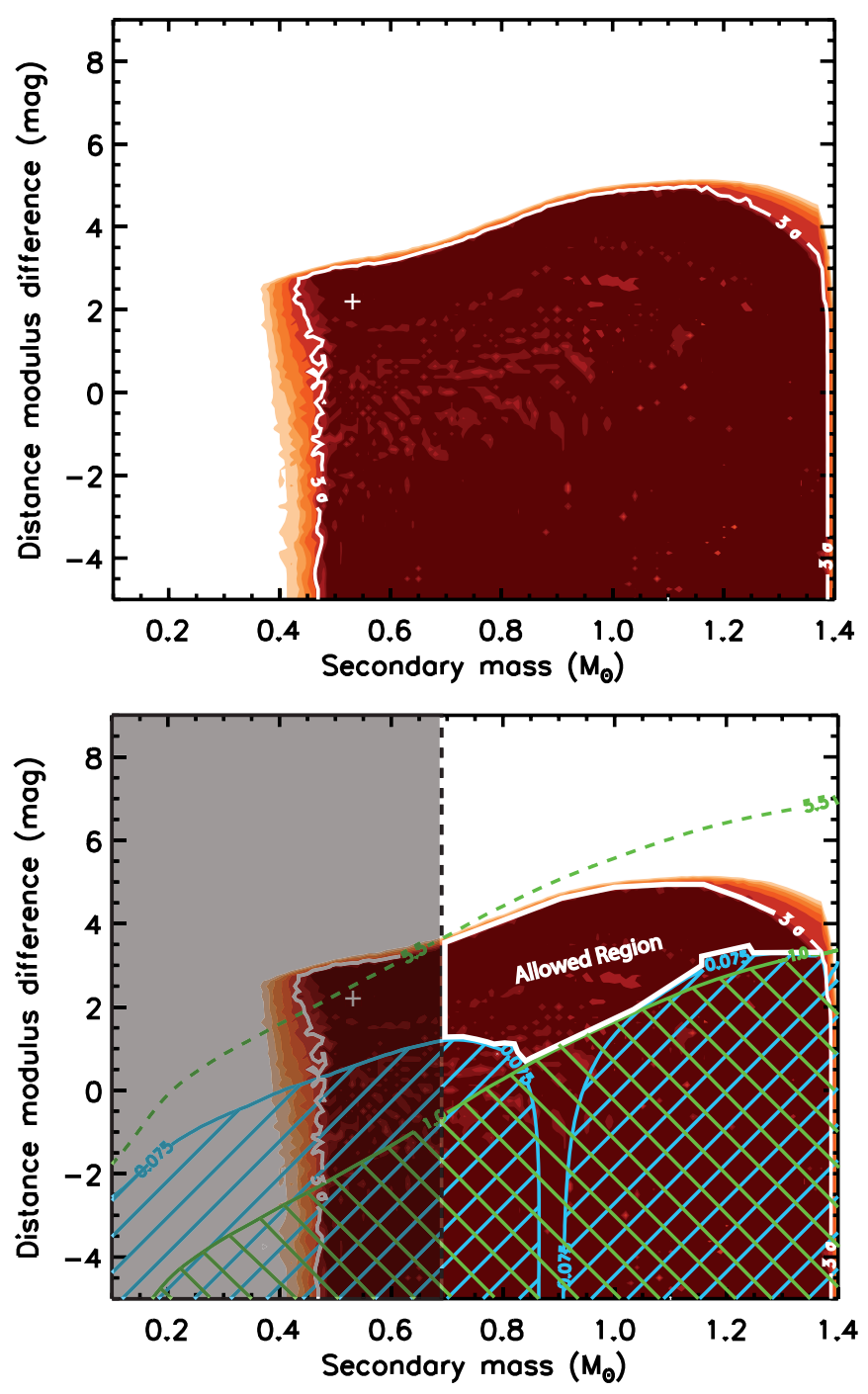

Figure 4. Top: map of the $\chi^{2}$ surface (goodness of fit) for blends involving background or foreground stars transited by a larger planet. The vertical axis represents the distance between the background pair of objects and the primary star, cast for convenience in terms of the difference in the distance modulus $\Delta \delta$ (note that $\Delta \delta$ is not equivalent to the magnitude difference between the background system and the main star because of the effects of differential extinction, which are included in our simulations). Only blends inside the solid white contour match the CoRoT light curve within acceptable limits ( $3 \sigma$, where $\sigma$ is the significance level of the $\chi^{2}$ difference; see Fressin et al. 2011). Lightercolored areas (red, orange, and yellow) mark regions of parameter space giving increasingly worse fits to the data $(4 \sigma, 5 \sigma$, etc.), and correspond to blends we consider to be ruled out. Bottom: same diagram as above with the addition of observational constraints from follow-up measurements. The constraint from our Spitzer observations is represented by the shaded gray area to the left of $0.69 M_{\odot}$; all false positives with secondary masses smaller than $0.69 M_{\odot}$ can be rejected, as they lead to transit depths at $4.5 \mu \mathrm{m}$ that are inconsistent with our measurements. Blends in the hatched green area are also ruled out because they are bright enough to be detected spectroscopically ( $\Delta m \leqslant 1.0 \mathrm{mag}$, represented by the solid green line). The hatched blue regions correspond to blends that can be excluded as well because of their $r-K_{s}$ colors, which are either too red (left) or too blue (right) compared to the measured value for CoRoT-7 by more than $3 \sigma(0.075 \mathrm{mag})$. The combination of all of these constraints leaves only a reduced area of parameter space (labeled "Allowed Region") where blend models give tolerable fits to the CoRoT light curve and are not ruled out by any of the follow-up observations. These blends are all brighter than $\Delta m=5.5 \mathrm{mag}$ (dashed green line). The white cross to the left of the Allowed Region marks the location of a representative blend ruled out by Spitzer, for which the predicted light curves are shown in Figure 5 (see the text).

(A color version of this figure is available in the online journal.)
Section 3 , this is a very conservative brightness limit as a large fraction of stars within 2 or even 3 mag of CoRoT-7 would most likely have been detected spectroscopically. As indicated before, our Spitzer observations exclude secondary stars less massive than approximately $0.69 M_{\odot}$, which are common constituents of background blends. This additional constraint is shown by the shaded gray area leftward of $0.69 M_{\odot}$. There is some overlap between the gray region and the blue and green hatched areas, indicating that those blends are excluded by more than one observational constraint.

An example of a blend that provides an acceptable fit to the CoRoT photometry is shown in Figure 5 (top panel). This scenario (location marked with a cross in Figure 4) is not excluded either by its color or its brightness, but is clearly ruled out by our Spitzer observations because it produces a much deeper transit in the near-infrared (see the bottom panel of Figure 5), which would have been easily detected. To summarize, the combination of the Spitzer, color, and brightness constraints removes many but not all blends involving a background or foreground star transited by a larger planet. Those that remain reside in the area of Figure 4 (bottom) labeled "Allowed Region."

\subsection{Background Eclipsing Binaries}

For the case of a background eclipsing binary composed of two stars, interestingly we find that no combination of relative distance and stellar properties for the eclipsing pair gives an acceptable fit to the CoRoT light curve. The reason for this is that all such blend configurations that can potentially reproduce the detailed shape of the transit also lead to out-of-eclipse brightness changes (ellipsoidal variations) with an amplitude that is not seen in the data, and that are a consequence of the very short orbital period. This implies that background blends of this kind can be confidently ruled out, an important conclusion that does not follow from the original Léger et al. (2009) analysis. Figure 6 shows that the blends that yield the best fits to the photometry are excluded at the $8.3 \sigma$ level or higher. This result is significant, as it substantially reduces the overall likelihood of blends for CoRoT-7 b.

We note that white dwarfs are also excluded as potential tertiaries. Although the range of their radii overlaps with those of the planets considered earlier in this section, their considerably larger mass would once again induce significant ellipsoidal variation in the light curve, which is ruled out by the observations. As was the case in the previous section, giant stars do not constitute viable secondaries because of the very short 0.8535 day period of the signal.

\subsection{Hierarchical Triples}

Finally, for eclipsing binaries (consisting of two stars) that are physically associated with the target in a hierarchical triple configuration, we find that the blend light curves invariably have the wrong shape to mimic a true transiting planet signal for any combination of stellar parameters for the secondary and tertiary. Either the depth, duration, or the steepness of the ingress/egress phases of the transits provides a very poor match to the CoRoT photometry, resulting in $\chi^{2}$ differences compared to the best transit model corresponding to several hundred $\sigma$. These scenarios are therefore all excluded. On the other hand, if we allow the tertiaries to be planets, the blend fits are somewhat better over a wide range of masses for the secondaries when transited by a planet of the appropriate size, 

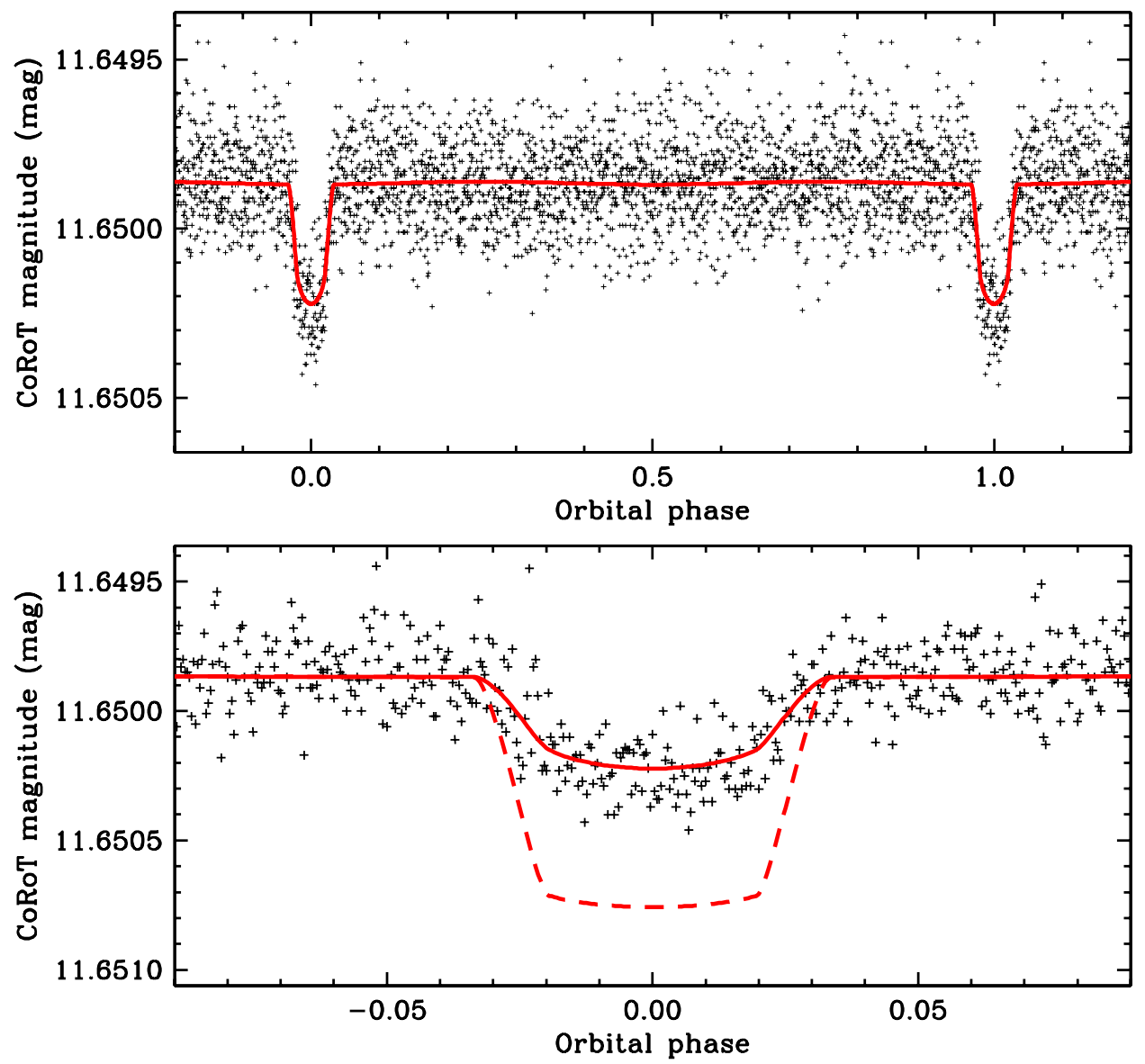

Figure 5. Example of a blend scenario (solid curves) that reproduces the visible light curve from CoRoT (black crosses), but that is ruled out solely by our Spitzer observations (and not by any of the other follow-up observations). It involves an M0 V star of $0.53 M_{\odot}$ with $V=17.0$ in the background of the target star, eclipsed by a Jovian planet with a radius 1.4 times that of Jupiter. Top: folded light curve, full phase. Bottom: enlargement around the transit of CoRoT-7 b. The dashed curve shows that the transit depth predicted for this same blend scenario in the near-infrared $4.5 \mu \mathrm{m}$ bandpass is much greater than in the optical, which is inconsistent with our findings (see also Section 2.1 and Figure 2).

(A color version of this figure is available in the online journal.)

but not quite at the $3 \sigma$ level or better. The best $\chi^{2}$ for a blend of this type corresponds to about a $3.4 \sigma$ departure from a planet model. Although our formal $3 \sigma$ limit is reasonable and consistent with common practice, it is still somewhat arbitrary and one may argue that fits that are only marginally worse might still be tolerable. Even accepting this possibility, Figure 7 shows that all of these blends are excluded by a combination of constraints from Spitzer, color index, and brightness, even those that are $10 \sigma$ or more away from the quality of a planet model.

\subsection{Summary of Blends}

From the simulations described above, the only viable blend scenarios for CoRoT-7 b are those involving a larger planet transiting a background or foreground star. BLENDER restricts those blends to the area labeled "Allowed Region" in Figure 4. These configurations involve stars between 1.0 and $5.5 \mathrm{mag}$ fainter than the target within the CoRoT aperture.

\section{VALIDATING CoRoT-7 b}

The a priori frequency of stars in the background or foreground of the target that are orbited by a transiting planet and are capable of mimicking the photometric signal may be estimated from the density of stars in the vicinity of CoRoT-7 and the

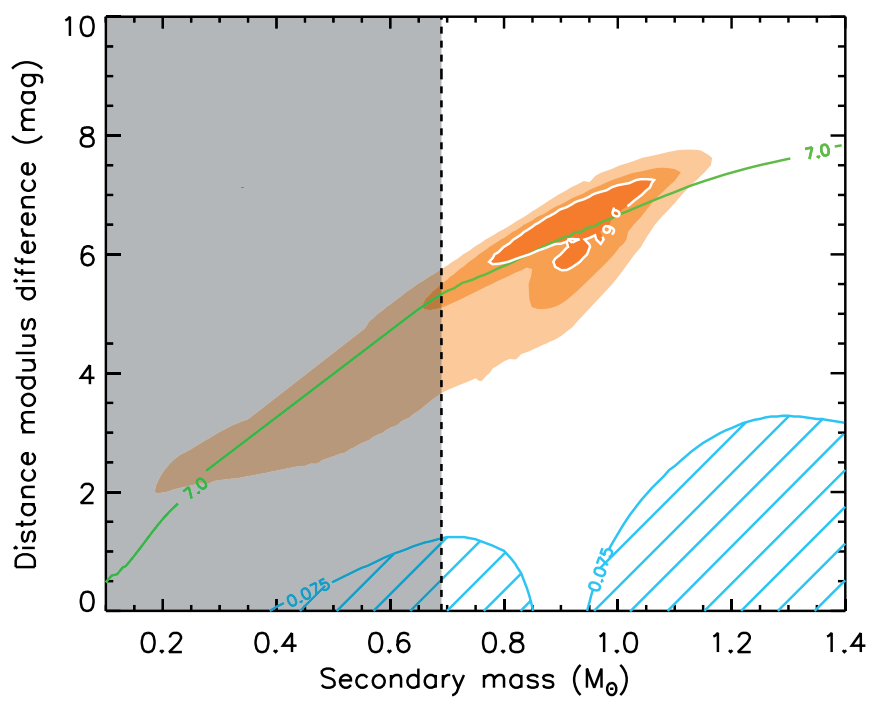

Figure 6. Similar to Figure 4 (and with the same color scheme) for blends involving background or foreground stars transited by another star. Using BLENDER we find that no such background eclipsing binary is able to reproduce the observed photometry at a level better than $8.3 \sigma$ (white contour) in comparison to the $\chi^{2}$ of the best planet fit. Therefore, this excludes all background or foreground eclipsing binaries consisting of two stars as potential false positives.

(A color version of this figure is available in the online journal.) 


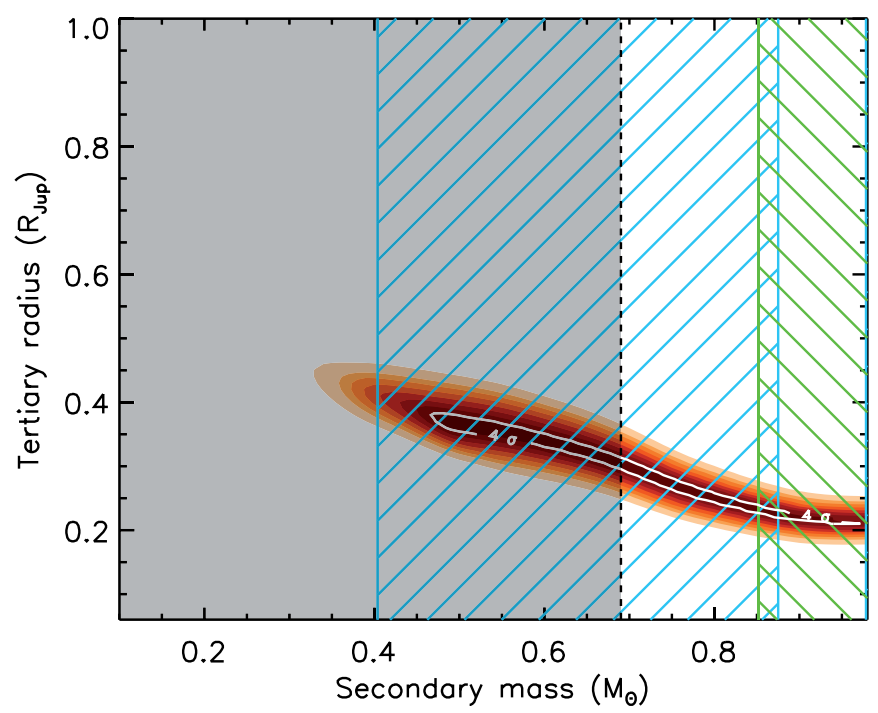

Figure 7. Similar to Figure 4 for the case of hierarchical triple systems in which the secondary is transited by a planet. After taking into account the Spitzer constraint (gray shaded region on the left), as well as the constraints on the $r-K_{s}$ color and those on the brightness of the secondaries from spectroscopy (blue and green hatched regions, respectively), we find that all triple configurations are excluded as false positives.

(A color version of this figure is available in the online journal.)

frequency of transiting planets with the appropriate characteristics. The relevant area around the target is that in which stars of each brightness would go undetected in the high-resolution imaging reported by the CoRoT team. To obtain the number density (stars per square degree) we make use of the Galactic structure models of Robin et al. (2003), and we perform this calculation in half-magnitude bins, as illustrated in Table 2. For each bin we further restrict the star counts using the constraints on the mass of the secondaries supplied by BLENDER (see Figure 4). These mass ranges are listed in Column 3 and the resulting densities appear in Column $4 .{ }^{12}$ Bins with no entries correspond to brightness ranges excluded by BLENDER. For the maximum angular separation $\left(\rho_{\max }\right.$, Column 5) at which stars of each brightness would escape detection we have adopted the value 0.4 , based on the report by Léger et al. (2009) that companions outside this range would have been seen in their VLT/NACO image down to a magnitude difference of $6.5 \mathrm{mag}$ compared to the target. We note that this $\rho_{\max }$ value is a conservative limit, as stars at closer separations would have been detected if they had smaller magnitude differences, which would reduce the BF. The result for the number of background stars in each magnitude bin is given in Column 6, in units of $10^{-6}$.

To estimate the frequency of transiting planets that might be expected to orbit these stars (and lead to a false positive) we rely on the results from Borucki et al. (2011), who reported a total of 1235 planet candidates among the 156,453 Kepler targets observed during the first four months of the mission. These signals have not yet been confirmed to be caused by planets and therefore remain candidates until they can be thoroughly followed up. However, the rate of false positives in this sample is expected to be quite small $(10 \%$ or less; see Morton \& Johnson 2011), so our results will not be significantly affected by the assumption that all of the candidates are planets. We

\footnotetext{
12 We note that the precipitous drop in the numbers listed in Column 4 below a magnitude of 16.7 is not due to a real decrease in stellar density (which in fact rises for fainter stars), but to the fact that the mass range allowed for blends in this magnitude bin is significantly reduced.
}

further assume that the census of Borucki et al. (2011) is largely complete. After accounting for the additional BLENDER constraint on the range of planet sizes for blends of this kind (tertiaries of $0.24-1.42 R_{\text {Jup }}$ ), we find that the transiting planet frequency $(\mathrm{PF})$ is $f_{\text {planet }}=571 / 156,453=0.0036$. Multiplying this frequency by the star counts in Column 6 of Table 2, we arrive at a total BF listed at the bottom of Column 7, $\mathrm{BF}=4.2 \times 10^{-7}$.

This figure represents the a priori likelihood of a false positive, and we note that it is approximately three orders of magnitude smaller than indicated by the calculations of Léger et al. (2009). However, we do not consider this to represent the "false alarm rate," as the expected likelihood of a transiting planet of the characteristics implied by the transit signal is also very small. We adopt here a Bayesian approach analogous to that employed to validate previous Kepler candidates, in which our confidence in the planetary nature of the signal will depend on how the blend likelihood compares to the a priori likelihood of a true transiting planet (PF) addressed below. Thus, we seek to estimate the odds ratio $\mathrm{PF} / \mathrm{BF}$. This is a significant conceptual difference compared to the frequentist approach by Léger et al. (2009), who considered only the likelihood of a blend (BF).

Implicit in the $\mathrm{BF}$ calculation above $\left(\mathrm{BF}=4.2 \times 10^{-7}\right)$ is the $3 \sigma$ criterion on the quality of the light curve fit relative to a transit model fit that we used as a condition for a blend scenario to be acceptable (see Section 4). For a fair comparison, we use a similar $3 \sigma$ criterion to establish the a priori transiting $\mathrm{PF}$ for the numerator of our odds ratio. We estimate PF by counting the Kepler candidates in the Borucki et al. (2011) sample that have radii within $3 \sigma$ of the value determined from the best fit to the CoRoT-7 data $\left(R_{p}=1.58 \pm 0.10 R_{\oplus}\right.$; Bruntt et al. 2010). We find 231 candidates within this range, giving a planet frequency $\mathrm{PF}=231 / 156,453=0.0015$.

Thus, the likelihood of a planet is more than 3500 times greater than that of a false positive $(\mathrm{PF} / \mathrm{BF}=0.0015 / 4.2 \times$ $10^{-7}$ ), which we consider sufficient to independently validate CoRoT-7 $\mathrm{b}$ as a true planet with a high degree of confidence. We note that our BF calculation assumes that the 1235 candidates cataloged by Borucki et al. (2011) are all true planets. If we were to assume conservatively that as many as $50 \%$ are false positives in the radius range specific to the transiting planet case (an unlikely proposition that is also inconsistent with other evidence; see Howard et al. 2010; Borucki et al. 2011), the planet likelihood would still be nearly 1800 times greater than the likelihood of a blend, implying a false alarm rate sufficiently small to validate the signal.

Additionally, in estimating the a priori true PF and the frequency of larger planets involved in blends, we have not placed any restriction on the periods of either kind of planet. One may expect, for example, that limiting the periods of larger planets involved in blends to be similar to that of CoRoT-7 b, which is quite short, might reduce the BF quite considerably. On the other hand, a similar period limit imposed on the true PF would decrease PF as well, though these effects may not be equal. Thus, it is possible that the odds ratio would be altered. As it turns out, our calculations without any period constraints are the most conservative. For example, limiting the periods to be shorter than five days, the census of Borucki et al. (2011) indicates that larger planets are comparatively less common than the smaller ones, resulting in an improvement in the PF/BF odds ratio of a factor of 2.2. Restricting the periods to be less than three days, the odds ratio improves by a factor of 2.8 . 
Table 2

Blend Frequency Estimate for CoRoT-7 b

\begin{tabular}{|c|c|c|c|c|c|c|}
\hline \multirow[b]{2}{*}{$\begin{array}{l}\text { Magnitude Range } \\
\text { (mag) }\end{array}$} & \multirow[b]{2}{*}{$\begin{array}{c}\Delta m \\
(\mathrm{mag})\end{array}$} & \multicolumn{5}{|c|}{ Blends Involving Planetary Tertiaries } \\
\hline & & $\begin{array}{l}\text { Stellar } \\
\text { Mass Range } \\
\left(M_{\odot}\right) \\
(3)\end{array}$ & $\begin{array}{l}\text { Stellar Density } \\
\text { (per sq. deg) }\end{array}$ & $\begin{array}{c}\rho_{\max } \\
(") \\
(5)\end{array}$ & $\begin{array}{c}\text { Stars } \\
\left(\times 10^{-6}\right) \\
(6)\end{array}$ & $\begin{array}{c}\text { Transiting Planets } \\
0.24-1.42 R_{\text {Jup }}, f_{\text {planet }}=0.36 \% \\
\left(\times 10^{-6}\right)\end{array}$ \\
\hline & & & & & & \\
\hline $11.7-12.2$ & 0.5 & $\ldots$ & $\ldots$ & $\ldots$ & $\ldots$ & $\ldots$ \\
\hline $12.2-12.7$ & 1.0 & $\ldots$ & $\ldots$ & $\ldots$ & $\ldots$ & $\ldots$ \\
\hline $12.7-13.2$ & 1.5 & $0.84-1.36$ & 91 & 0.40 & 3.53 & 0.013 \\
\hline $14.2-14.7$ & 3.0 & $0.69-1.26$ & 263 & 0.40 & 10.2 & 0.037 \\
\hline $14.7-15.2$ & 3.5 & $0.69-1.22$ & 383 & 0.40 & 14.9 & 0.054 \\
\hline $15.2-15.7$ & 4.0 & $0.69-1.17$ & 515 & 0.40 & 20.0 & 0.072 \\
\hline $15.7-16.2$ & 4.5 & $0.69-1.08$ & 569 & 0.40 & 22.0 & 0.081 \\
\hline $16.2-16.7$ & 5.0 & $0.69-0.99$ & 637 & 0.40 & 24.7 & 0.090 \\
\hline $16.7-17.2$ & 5.5 & $0.69-0.80$ & 186 & 0.40 & 7.21 & 0.026 \\
\hline $19.2-19.7$ & 8.0 & $\cdots$ & $\cdots$ & $\cdots$ & $\ldots$ & $\cdots$ \\
\hline $19.7-20.2$ & 8.5 & $\begin{array}{l}\cdots \\
\ldots\end{array}$ & $\cdots$ & $\cdots$ & $\cdots$ & $\cdots$ \\
\hline Totals & & & 2988 & & 115.9 & 0.42 \\
\hline
\end{tabular}

Total frequency $(\mathrm{BF})=4.2 \times 10^{-7}$

Note. Magnitude bins with no entries correspond to brightness ranges in which BLENDER excludes all blends.

\section{DISCUSSION}

Our observation of four transits of CoRoT-7 b with the Spitzer Space Telescope at a wavelength of $4.5 \mu \mathrm{m}$ has resulted in the detection of the transit with a depth of $0.426 \pm 0.115 \mathrm{mmag}$, which is consistent with the CoRoT-7 b planet scenario described in Léger et al. (2009). Although the signal-to-noise ratio of this infrared detection is relatively low, it has been obtained with a now standard treatment of the Spitzer data with no a priori knowledge of the transit parameters (aside from the timing windows in order to plan the four Spitzer visits).

Warm Spitzer is currently the only facility available that has the capability of detecting such shallow transits at wavelengths that are sufficiently separated from the CoRoT (or Kepler) passbands to be helpful. In this case, the observations were successful and the transit at $4.5 \mu \mathrm{m}$ is shown to have virtually the same depth as in the optical. This places a strong constraint on the color of potential blends, which are restricted to have secondaries of similar spectral type as the primary star. In the case of CoRoT-7 b this allows us to rule out most cool stars (which constitute the majority of background stars) as potential contaminants.

The detailed analysis of the CoRoT-7 photometry with BLENDER combined with constraints from other observations eliminates the vast majority of possible blend scenarios. This includes all background eclipsing binaries composed of two stars, most of the scenarios involving chance alignments with a star transited by a larger planet, and all hierarchical triple configurations. The remaining scenarios are much less likely (by a factor of 3500) than a true transiting planet, thereby validating the planetary nature of the signal with very high confidence. We point out that this conclusion has been reached with very conservative assumptions regarding some of the observa- tional constraints. For example, we have ignored the fact that the high-resolution VLT/NACO imaging by Léger et al. (2009) permits the detection of background stars closer than our adopted limit of $\rho_{\max }=0^{\prime \prime} 4$ from the target if they are relatively bright. Additional imaging by those authors with FASTCAM on the $2.5 \mathrm{~m}$ NOT telescope provides even tighter constraints that we have not used, reaching down to sensitivities of 4 mag fainter than the target at $0{ }^{\prime \prime} 18$. We have also not considered the full potential of spectroscopy to rule out closer companions; we have assumed only that stars within 1.0 mag of the target would have been identified, whereas in reality the sensitivity of those observations (not only the near-infrared CRIRES spectrum, but also those from HARPS) is probably much greater $(\Delta m \approx$ 2-3 mag). Furthermore, we have not made use of other information reported by Léger et al. (2009), such as the constraints from the red, green, and blue passbands into which the light from the CoRoT instrument can be split, which can further limit the pool of potential false positives. Incorporating all of these constraints can only reduce the $\mathrm{BF}$, resulting in an even greater confidence level for the validation of CoRoT-7 b as the first super-Earth.

Our Spitzer observations have also significantly improved our knowledge of the ephemeris of the first transiting superEarth. Transits of CoRoT-7 b have not been observed with any other photometric facility since the end of the LRa01 field observations by the CoRoT satellite. Given the formal uncertainty in the orbital period reported by Léger et al. (2009), the accumulated error in the predicted times of transit up to the date of our Spitzer observations is approximately 20 minutes, or about a quarter of the transit duration. Here we have improved the period determination by about a factor of four, to $P=0.853590 \pm 0.000006$ days, ensuring that the transits will be recoverable for at least another decade (provided 
there are no physical mechanisms operating to change the period).

The Kepler satellite has recently released a large number of very promising shallow transit candidates (Borucki et al. 2011). From the high quality of this photometry the detailed transit shapes are often very well defined, increasing the power of tools such as BLENDER that make use of that information to rule out blend scenarios that result in poor fits to the data. The exquisite relative astrometric precision delivered by the Kepler instrument provides important additional constraints from an analysis of the motion of the photocenters of the images in and out of transit (see, e.g., Batalha et al. 2011), which can typically exclude $90 \%$ or more of unresolved stars falling within the photometric aperture that can potentially contaminate the flux measurements. Spectroscopy and high-resolution imaging contribute further valuable constraints. These tools have already been used to validate small signals for which radial-velocity confirmation is currently out of reach, including possibly rocky planets. Examples include the super-Earth Kepler-9 d (Torres et al. 2011), and the Neptune-size planets Kepler-10 c (Fressin et al. 2011), Kepler-11 g (Lissauer et al. 2011), and Kepler-19 b (Ballard et al. 2011).

Spitzer observations provide a very effective way of ruling out a large fraction of the blend scenarios involving a background star with a different effective temperature (or color) than the target. These chance alignments are typically the most serious concern regarding the nature of the transit signals. Among the most interesting candidates expected from the Kepler Mission are rocky planets in the habitable zone of their parent stars. The typically longer periods of these objects mean that methods of confirmation relying on the dynamical influence of the planet and/or the quality of the phase-folded photometry (obtained by summing individual transit events) will be more problematic, as their efficiency scales down with orbital period. We anticipate that the Spitzer telescope in its warm phase will prove critical for validating such objects, as its efficiency does not depend on period, but relies instead on a different intrinsic property of the transits, which is their achromaticity.

We are grateful to the anonymous referee for many very helpful comments and suggestions. This work is based on observations made with the Spitzer Space Telescope, which is operated by the Jet Propulsion Laboratory, California Institute of Technology, under contract to NASA. Support for this work was provided by NASA through an award issued by JPL/Caltech. This research has made use of the facilities at the NASA Advanced Supercomputing Division (NASA Ames Research Center).

\section{REFERENCES}

Agol, E., Cowan, N. B., Knutson, H. A., et al. 2010, ApJ, 721, 1861

Aigrain, S., \& Irwin, M. 2004, MNRAS, 350, 331

Baglin, A., Auvergne, M., Barge, P., et al. 2002, in Proc. Conf. on Stellar Structure and Habitable Planet Finding, Vol. 485, ed. B. Battrick, F. Favata, I. W. Roxburgh, \& D. Galadi (ESA SP-485; Noordwijk: ESA), 17

Ballard, S., Fabrycky, D., Fressin, F., et al. 2011, ApJ, in press (arXiv:1109.1561)

Batalha, N. M., Borucki, W. J., Bryson, S. T., et al. 2011, ApJ, 729, 27

Berta, Z. K., Charbonneau, D., Bean, J., et al. 2011, ApJ, 736, 12

Boisse, I., Bouchy, F., Hébrard, G., et al. 2011, A\&A, 528, 4

Borucki, W. J., Koch, D. G., Basri, G., et al. 2011, ApJ, 736, 19

Bruntt, H., Deleuil, M., Fridlund, M., et al. 2010, A\&A, 519, A51

Charbonneau, D., Allen, L. E., Megeath, S. T., et al. 2005, ApJ, 626, 523

Charbonneau, D., Berta, Z. K., Irwin, J., et al. 2009, Nature, 462, 891

Charbonneau, D., Knutson, H. A., Barman, T., et al. 2008, ApJ, 686, 1341

Claret, A. 2000, A\&A, 363, 1081

Fazio, G. G., Hora, J. L., Allen, L. E., et al. 2004, ApJS, 154, 10

Ferraz-Mello, S., Tadeu dos Santos, M., Beaugé, C., Michtchenko, T. A., \& Rodrí1guez, A. 2011, A\&A, 531, 161

Ford, E. B. 2005, AJ, 129, 1706

Fressin, F., Guillot, T., \& Nesta, L. 2009, A\&A, 504, 605

Fressin, F., Torres, G., Desert, J. M., et al. 2011, ApJS, 197, 5

Girardi, L., Bressan, A., Bertelli, G., \& Chiosi, C. 2000, A\&AS, 141, 371

Hatzes, A. P., Dvorak, R., Wuchterl, G., et al. 2010, A\&A, 520, A93

Hatzes, A. P., Fridlund, M., Nachmani, G., et al. 2011, arXiv:1105.3372

Howard, A. W., Marcy, G. W., Johnson, J. A., et al. 2010, Science, 330, 653

Knutson, H. A., Charbonneau, D., Allen, L. E., Burrows, A., \& Megeath, S. T. 2008, ApJ, 673, 526

Knutson, H. A., Charbonneau, D., Burrows, A., et al. 2009, ApJ, 655, 566

Léger, A., Rouan, D., Schneider, J., et al. 2009, A\&A, 506, 287

Lissauer, J. J., Fabrycky, D. C., Ford, E. B., et al. 2011, Nature, 470, 53

Mandel, K., \& Agol, E. 2002, ApJ, 580, L171

Markwardt, C. B. 2009, in ASP Conf. Ser. 411, Astronomical Data Analysis Software and Systems XVIII, ed. D. A. Bohlender, D. Durand, \& P. Dowler (San Francisco, CA: ASP), 251

Mazeh, T. 2008, in Tidal Effects in Stars, Planets and Disks, Vol. 29 ed. M.-J. Goupil \& J.-P. Zahn (EAS Publications Series; EDP Sciences), 1

Morales-Calderon, M., Stauffer, J. R., Kirkpatrick, J. D., et al. 2006, ApJ, 653, 1454

Morton, T. D., \& Johnson, J. A. 2011, ApJ, 738, 170

Pont, F., Aigrain, S., \& Zucker, S. 2011, MNRAS, 411, 1953

Pont, F., Zucker, S., \& Queloz, D. 2006, MNRAS, 373, 231

Queloz, D., Bouchy, F., Moutou, C., et al. 2009, A\&A, 506, 303

Reach, W. T., Megeath, S. T., Cohen, M., et al. 2005, PASP, 117, 978

Robin, A. C., Reylé, C., Derriére, S., \& Picaud, S. 2003, A\&A, 409, 523

Sing, D. K. 2010, A\&A, 510, 21

Torres, G., Fressin, F., Batalha, N. M., et al. 2011, ApJ, 727, 24

Torres, G., Konacki, M., Sasselov, D. D., \& Jha, S. 2004, ApJ, 614, 979

Werner, M. W., Roellig, T. L., Low, F. J., et al. 2004, ApJS, 154, 1

Winn, J. N., Holman, M. J., \& Fuentes, C. I. 2007, AJ, 133, 11

Winn, J. N., Johnson, J. A., Albrecht, S., et al. 2009, ApJ, 703, L99 